\title{
Chameleon mechanism on gravitational wave in $f(R)$ gravity
}

\section{Tomohiro Nakamura $^{* a}$, Taishi Katsuragawa ${ }^{b}$, Taishi Ikeda ${ }^{c}$ and Salvatore Capozziello $^{\text {def }}$}

${ }^{a}$ Department of Physics, Nagoya University, Nagoya 464-8602, Japan

${ }^{b}$ Institute of Astrophysics, Central China Normal University, Wuhan 430079, China

${ }^{c}$ CENTRA, Departamento de Física, Instituto Superior Técnico - IST, Universidade de LisboaUL, Avenida Rovisco Pais 1, 1049 Lisboa, Portugal

${ }^{d}$ Dipartimento di Fisica "E. Pancini", Università di Napoli "Federico II", Complesso

Universitario di Monte Sant' Angelo, Edificio G, Via Cinthia, I-80126, Napoli, Italy

eIstituto Nazionale di Fisica Nucleare (INFN) Sezione di Napoli, Complesso Universitario di Monte Sant' Angelo, Edificio G, Via Cinthia, I-80126, Napoli, Italy

${ }^{f}$ Laboratory for Theoretical Cosmology, Tomsk State University of Control Systems and

Radioelectronics (TUSUR), 634050 Tomsk, Russia.

E-mail: nakamura.tomohiroeg.mbox.nagoya-u.ac.jp

taishi@mail.ccnu.edu.cn taishi.ikedadtecnico.ulisboa.pt

capozzielloena.infn.it

\begin{abstract}
We investigate how the so-called chameleon mechanism in $F(R)$ gravity acts on the scalar mode of gravitational waves appearing as an additional polarization mode to usual tensor modes in general relativity. We calculate its amplitude in a simplified situation imitating the ground-based gravitational wave detectors and estimate the detectability. Our conclusion is that it is almost impossible to detect scalar mode due to the chameleon mechanism and space-based gravitational wave detector would be a significant tool to detect such additional modes.
\end{abstract}

The 4th KMI International Symposium (KMI2019)

18-20, February 2019

Nagoya, Japan

\footnotetext{
${ }^{*}$ Speaker.
} 


\section{Introduction}

Gravitational waves (GWs) are now an important tool to investigate the possibility of alternative gravitational theories. One of the important property completely different from general relativity for alternative theories is the number of polarization modes. If we take $F(R)$ gravity, which has an action of an arbitrary function of the Ricci scalar instead of Einstein-Hilbert action, as an example, it contains an additional scalar mode to usual tensor ones. Simultaneous observation of GWs by multiple detectors are expected to give the information about the existence of the extra polarization mode in near future. However, $\mathrm{F}(\mathrm{R})$ gravity has a so-called chameleon mechanism to evade the experimental constraint on the fifth force due to the existence of an additional scalar degree of freedom. In our work, we investigate the detectability of the scalar mode with considering the chameleon mechanism.

\section{2. $F(R)$ gravity and chameleon mechanism}

The action of the generic $F(R)$ gravity model is given as follows:

$$
S=\frac{1}{2 \kappa^{2}} \int d^{4} x \sqrt{-g} F(R)+\int d^{4} x \sqrt{-g} \mathscr{L}_{\text {Matter }}\left[g^{\mu \nu}, \Psi\right]
$$

where $F(R)$ is a function of the Ricci scalar $R$ and $\kappa^{2}=8 \pi G=1 / M_{\mathrm{pl}}^{2} \cdot M_{\mathrm{pl}}$ is the reduced Planck mass, and $M_{\mathrm{pl}} \simeq 2 \times 10^{18}\left[\mathrm{GeV}^{2}\right]$. $\mathscr{L}_{\text {Matter }}$ denotes the Lagrangian for a matter field $\Psi$. We obtain the equations of motion by taking the variation of the action (2.1) with respect to the metric $g_{\mu \nu}$ as follows,

$$
F_{R}(R) R_{\mu v}-\frac{1}{2} F(R) g_{\mu v}+\left(g_{\mu v} \square-\nabla_{\mu} \nabla_{v}\right) F_{R}(R)=\kappa^{2} T_{\mu v}\left(g^{\mu v}, \Psi\right) .
$$

$F_{R}(R)$ expresses the derivative of $F(R)$ with respect to $R, F_{R}(R)=\partial_{R} F(R)$. The energy-momentum tensor $T_{\mu \nu}$ is defined by $T_{\mu \nu}\left(g^{\mu v}, \Psi\right)=-2 / \sqrt{-g} \delta\left(\sqrt{-g} \mathscr{L}_{\text {Matter }}\left(g^{\mu v}, \Psi\right)\right) / \delta g^{\mu \nu}$. Taking the trace of the equations (2.2), we obtain

$$
\square F_{R}(R)=\frac{1}{3}\left[2 F(R)-R F_{R}(R)+\kappa^{2} T\right]
$$

where the Ricci scalar $R$ and the trace of the energy-momentum tensor $T$ are given by $R=R_{\mu}^{\mu}$ and $T=T_{\mu}^{\mu}$ respectively.

We can define scalar field by the following identification: $\Phi \equiv F_{R}(R)$. Solving the above relation with respect to $\Phi$, one can express the Ricci curvature $R$ in terms of $\Phi, R=R(\Phi)$. Then, we can regard Eq. (2.3) as the equation motion for an additional scalar degree of freedom,

$$
\square \Phi=\frac{\mathrm{d} V_{\mathrm{eff}}(\Phi)}{\mathrm{d} \Phi}
$$

where we define the effective potential $V_{\text {eff }}$ by $\mathrm{d} V_{\text {eff }} / \mathrm{d} \Phi=\frac{1}{3}\left[2 F(R)-R F_{R}(R)+\kappa^{2} T\right]$. We can understand the general behavior of the scalar field by expanding Eq. (2.4) from the potential minimum 
$\Phi=\Phi_{\min }$, which is obtained by solving the stationary condition $\mathrm{d} V_{\text {eff }} / \mathrm{d} \Phi=0$. The second derivative of the effective potential can be regarded as the mass of the scalar field. Defining $R=R_{\min }$ satisfying $\Phi_{\min }=F_{R}\left(R_{\min }\right)$, we obtain

$$
m_{\Phi}^{2}=\left.\frac{\mathrm{d}^{2} V_{\mathrm{eff}}(\Phi, T)}{\mathrm{d} \Phi^{2}}\right|_{\Phi=\Phi_{\min }}=\frac{1}{3}\left[\frac{F_{R}\left(R_{\min }\right)}{F_{R R}\left(R_{\min }\right)}-R_{\min }\right] .
$$

It is the key feature of the chameleon mechanism that the effective potential $V_{\text {eff }}$ contains the trace of energy-momentum tensor $T$. Since the potential minimum changes according to the matter distribution, the mass of scalar field Eq. (2.5) acquires the environment dependence.

It is notable that Eq. (2.2) can have the de Sitter solution $R_{\mu \nu}=\Lambda g_{\mu v}$, if the following relation satisfies:

$$
2 F(R)-R F_{R}(R)+\kappa^{2} T=0
$$

which exactly corresponds to the stationary condition or potential minimum of the effective potential $V_{\text {eff }}$. Thus, the $F(R)$ gravity can be a solution to the dark energy problem giving naturally rise to the cosmological constant [1].

In the chameleon mechanism, the scalar field potential depends on the energy-momentum tensor of the other matter fields, which results in that the mass of the scalar field changes according to the information of the surrounding matter fields. If we choose the potential so that the mass increases at the high-density region and decreases at the low-density region, the chameleonic scalar field can have the potential to explain the experiments in two different scales: In cosmological scale, it behaves as a light scalar field accelerating the expansion of the universe. On the other hand, in smaller scale, it has a large mass so that it does not affect any result of the gravitational experiments.

As an example, we consider a concrete model of $F(R)$ gravity for dark energy which is free from the curvature singularity problem[2, 3, 4]:

$$
F(R)=R-\beta R_{c}\left[1-\left(1+\frac{R^{2}}{R_{c}^{2}}\right)^{-n}\right]+\alpha R^{2},
$$

In the large-curvature limit, we obtain the mass of scalar field at the potential minimum:

$$
m_{\Phi}^{2} \approx \frac{1}{3}\left[\frac{1-2 n \beta\left(\frac{R_{c}}{R_{\min }}\right)^{2 n+1}+2 \frac{\mu}{R_{c}} R_{\min }}{\frac{2 n(2 n+1) \beta}{R_{c}}\left(\frac{R_{c}}{R_{\min }}\right)^{2 n+2}+2 \frac{\mu}{R_{c}}}-R_{\min }\right] .
$$

where $R_{\min }$ is determined by the energy-momentum tensor by $R_{\min } \approx-\kappa^{2} T$. The detailed derivation is given in [5]. When we use the pressure-less dust for the matter fields, the trace of the energy-momentum tensor is given as $T=-\rho$ where $\rho$ is the density of the matter, which gives the density dependence to the mass through the curvature as $R_{\min }=\kappa^{2} \rho$. Because the mass 2.8) is the increasing function of the curvature, we can conclude that the scalar field becomes heavy in the high-density region. 


\section{Gravitational wave in $F(R)$ gravity}

Next, we consider the gravitational wave in $\mathrm{F}(\mathrm{R})$ gravity as a perturbation from the background metric. We define the perturbation for the metric $g_{\mu \nu}$ and scalar field $\Phi$ :

$$
\begin{aligned}
g_{\mu v} & =b_{\mu v}+h_{\mu v}, \\
\Phi & =\Phi_{\min }+\phi,
\end{aligned}
$$

where $b_{\mu v}$ is the background metric, and $\Phi_{\min }$ satisfies $d V_{\text {eff }} / d \Phi=0$.

For simplicity, we assume that the background is maximally symmetric, that is, the Minkowski and (anti-)de Sitter background. Then, we obtain the equations for the perturbation at the first order:

$$
\begin{aligned}
\delta R_{\mu v}-\frac{1}{2} \delta R b_{\mu v}-\frac{1}{2} \frac{F\left(R^{(b)}\right)}{\Phi_{\min }} h_{\mu v} & =\left(\nabla_{\mu}^{(b)} \nabla_{v}^{(b)}-b_{\mu v} \square^{(b)}-R_{\mu v}^{(b)}\right) \phi, \\
\left(\square-m_{\Phi}^{2}\right) \varphi & =-\frac{1}{3} \Phi_{\min } h^{\mu v} R_{\mu v}^{(b)}+\frac{1}{6} F\left(R^{(b)}\right) h
\end{aligned}
$$

where we define the new scalar field perturbation $\varphi \equiv \phi / \Phi_{\min }$, to normalize the original perturbation $\phi$.

In the dark energy $F(R)$ models, we cannot take the Minkowski background. However, since the curvature of the background varies at cosmological scale, we can safely ignore the curvature term and obtain the same equation in the Minkowski background as long as we consider much smaller scale than cosmological one.

\section{Environment dependence of scalar wave}

Next, we solve Eq. 3.4 in a simple case. We consider the situation where the plane scalar wave is propagating form infinitely far region to spherical high-density region. For more simplification, we focus on the region close to the surface of the high density region where the scalar wave enters almost vertically. The other situations are considered in [5]. For this assumption, we can approximate the situation as the plane scalar wave propagates low-density region to high-density region in $(1+1)$ dimension. if we denote the propagating direction of the scalar mode of GWs by $z$ coordinate and the position of the boundary between the high-density region and space as $z=z_{b}\left(=r_{b}\right), \Phi_{b g}$ can be approximated as follows:

$$
\Phi_{b g}(z)=\left\{\begin{array}{cc}
\Phi_{a} & \left(|z| \leq z_{b}\right) \\
\Phi_{\infty}-\left(\Phi_{\infty}-\Phi_{a}\right) z_{b} \frac{e^{-m_{\infty}\left(z-z_{b}\right)}}{z} & \left(z_{b}<|z|\right)
\end{array}\right.
$$

where subscripts $a$ and $\infty$ means the potential minimum value for $\rho=\rho_{a}, \rho_{\infty}$ respectively. Substituting it into Eq. (3.4), one obtains the equation of motion for the single frequency scalar wave:

$$
\frac{\mathrm{d}^{2} \tilde{\phi}}{\mathrm{d} z^{2}}=\left(m_{\Phi}^{2}\left(\Phi_{b g}\right)-\omega^{2}\right) \tilde{\phi}
$$

where means the Fourier expansion coefficient of $\phi$. If we regard the right-hand side of the equation as a potential, Eq. (4.2) takes the form of the wave equation with the potential $V(z) \equiv$ $m_{\Phi}^{2}\left(\Phi_{b g}\right)$. 
Finally, we can make a rough estimation for an amplitude of the scalar wave in the highdensity region. For scalar waves with the frequency $\omega<m_{\Phi}$, the solution of Eq. (4.2) inside of the high-density region becomes

$$
\tilde{\phi}=C e^{\sqrt{m_{\Phi}^{2}-\omega^{2}} z},
$$

where $C$ is a constant which represents the amplitude of the scalar wave, and we took only one mode of the solution in order to satisfy the boundary condition at the center. As an illustration, if we choose a concrete model 2.7) with fixing the parameters as $\beta=2, n=1$ and $\mu=10^{-62}$ and assume the energy density in the atmosphere $\rho=\rho_{a}=10^{-9}\left[\mathrm{~g} / \mathrm{cm}^{3}\right]$ at around $10^{5}[\mathrm{~m}]$ of the altitude, we obtain

$$
m_{a} \simeq 8 \times 10^{-12}[\mathrm{GeV}]
$$

Therefore, the suppression factor is roughly estimated as

$$
\exp \left[-\sqrt{m_{a}^{2}-\omega^{2}}|z|\right] \sim \exp \left[-m_{a}|z|\right] \simeq 10^{-6 \times 10^{9}} .
$$

The scalar waves which have a lower frequency than criterion frequency determined by the mass in the atmosphere receive huge suppression at the detection. We also note that the criterion frequency of the suppression can be calculated from $\omega_{c}=m_{a}$ by the unit conversion as $\omega_{c} \simeq 1 \times 10^{13}[\mathrm{~Hz}]$, which is much higher than the current typical frequency of GWs detected by LIGO and Virgo.

Furthermore, the typical amplitude of the possible scalar mode by a binary system can be estimated by the orbital period change of Hulse-Taylor binary [6] as

$$
C \sim o(0.01) \times A_{\text {tensor }}
$$

where $A_{\text {tensor }}$ is a typical amplitude of the tensor mode from a binary system. At the detection in grand-based detectors, the huge suppression factor should be multiplied to the amplitude. Therefore, it is far beyond the detectable band of the current observational sensitivity.

\section{Conclusion}

We have investigated the detectability of the scalar mode in $F(R)$ gravity focusing on the chameleon mechanism. We have found it is almost impossible to detect such modes by grandbased GW detectors due to the huge suppression in the atmosphere. However, currently planning space-based detectors, such as eLISA and DECIGO, have the possibility to detect additional modes since it is not affected by the chameleonic suppression of the atmosphere.

\section{References}

[1] S. Capozziello, Int. J. Mod. Phys. D 11 (2002) 483

[2] A. V. Frolov, Phys. Rev. Lett. 101, 061103 (2008)

[3] A. Dev, D. Jain, S. Jhingan, S. Nojiri, M. Sami and I. Thongkool, Phys. Rev. D 78, 083515 (2008)

[4] T. Kobayashi and K. Maeda, Phys. Rev. D 79, 024009 (2009)

[5] T. Katsuragawa, T. Nakamura, T. Ikeda and S. Capozziello, arXiv:1902.02494 [gr-qc]

[6] C. M. Will, Living Rev. Rel. 17, 4 (2014). 